\title{
Preparação, sinterização e espectroscopia de impedância da zircônia parcialmente estabilizada com cálcia
}

\author{
(Synthesis, sintering and impedance spectroscopy of calcia-partially \\ stabilized zirconia)
}

\author{
E. N. S. Muccillo, R. C. Buissa Neto, S. K. Tadokoro, R. Muccillo \\ Centro Multidisciplinar para o Desenvolvimento de Materiais Cerâmicos \\ CCTM - Instituto de Pesquisas Energéticas e Nucleares \\ C. P. 11049, S. Paulo, SP 05422-970 \\ enavarro@usp.br
}

\begin{abstract}
Resumo
Cerâmicas de zircônia parcialmente estabilizada com cálcia foram preparadas com pós nanométricos obtidos pela técnica da co-precipitação. A sinterização dos compactos foi feita a $1500{ }^{\circ} \mathrm{C}$ com tempos de patamar de 3 min e 2 h para o estudo do efeito produzido por diferentes fases na resistividade elétrica da cerâmica sinterizada. O estudo da decomposição térmica do gel mostrou que a formação do óxido ocorre a temperaturas relativamente baixas. A área de superfície específica é elevada quando comparada com valores dos materiais comerciais. A densificação de compactos preparados com pós nanométricos inicia a temperaturas inferiores a $1000{ }^{\circ} \mathrm{C}$, mas a cinética de sinterização é dependente da fase formada durante o aquecimento. Os compactos sinterizados apresentam fases distintas dependendo do tempo de sinterização à mesma temperatura. A contribuição relativa das fases formadas na zircônia parcialmente estabilizada para a resistividade elétrica das cerâmicas sinterizados pode ser diferenciada por meio de medidas de espectroscopia de impedância.

Palavras-chave: espectroscopia de impedância, zircônia, síntese de pós, sinterização.
\end{abstract}

\begin{abstract}
$\mathrm{CaO}$-partially stabilized $\mathrm{ZrO}_{2}$ ceramics were prepared from nanosized powders synthesized by the coprecipitation technique. In order to study the effect of different crystallographic phases on the electrical resistivity, powder compacts were sintered at $1500{ }^{\circ} \mathrm{C}$ for soaking times of $2 \mathrm{~h}$ and $3 \mathrm{~min}$. The thermal decomposition of the precursor gel occurs at low temperatures giving rise to a high specific surface area powder material. The densification of powder compacts starts at temperatures lower than $1000^{\circ} \mathrm{C}$, although the sintering kinetics depends on the crystallographic phase formed during heating of the compact. The phase assemblage of sintered compacts depends on the soaking time. Impedance spectroscopy measurements allowed for identification of the relative contribution of different phases to the total electrical resistivity of calcia-partially stabilized zirconia ceramics.

Keywords: impedance spectroscopy, zirconia, powder synthesis, sintering.
\end{abstract}

\section{INTRODUÇÃO}

Cerâmicas à base de zircônia são muito utilizadas principalmente por suas propriedades elétricas e mecânicas. Em termos de propriedades elétricas, esses materiais exibem alta condutividade iônica a altas temperaturas $\left(>500{ }^{\circ} \mathrm{C}\right.$ ) o que permite seu uso em sensores de oxigênio, células a combustível de eletrólito sólido óxido, e outros dispositivos nos quais esta propriedade seja requerida $[1,2]$.

A condutividade, $\sigma$ (ou resistividade, $\rho=1 / \sigma$ ), iônica nessas cerâmicas está diretamente relacionada com sua estrutura cristalina, composição química e de fases. Por isso, diversos trabalhos encontrados na literatura foram dedicados ao estudo da correlação entre estrutura e resistividade elétrica, destacando-se aqueles das referências [3-9].
Do ponto de vista de aplicações tecnológicas, a zircônia dopada com cálcia é o material preferido para a confecção de tubos que são amplamente utilizados em células eletroquímicas, além de bocais para escoamento de metais fundidos [10-12].

Apesar da existência de diversos diagramas de fase para a solução sólida entre $\mathrm{ZrO}_{2}$ e $\mathrm{CaO}$, aquele mostrado na Fig. 1 da ref. [13] é um dos mais citados. Na faixa de altas temperaturas o diagrama de fases exibe diversas regiões de interesse. A primeira consiste de uma solução sólida com estrutura tetragonal, $\mathrm{t}$ (até $\sim 5,5 \% \mathrm{~mol} \mathrm{CaO}$ ), sendo termodinamicamente estável entre $\sim 1200{ }^{\circ} \mathrm{C}$ e $\sim 2300{ }^{\circ} \mathrm{C}$. A segunda região envolve o campo de fases misto cúbico, c, mais tetragonal, para temperaturas superiores a $1140{ }^{\circ} \mathrm{C}$. Esta região se estende de $\sim 6 \mathrm{a} \sim 12,5 \% \mathrm{~mol} \mathrm{CaO}$. A terceira 
região de interesse é aquela que compreende a fase cúbica termodinamicamente estável em composições acima de $12,5 \%$ mol de $\mathrm{CaO}$, a temperaturas superiores a $1140{ }^{\circ} \mathrm{C}$. Um aspecto importante neste diagrama de fase são os extensos campos de estabilidade das fases tetragonal mais cúbica, e cúbica. Disto resulta que cerâmicas parcialmente ou totalmente estabilizadas podem ser obtidas em amplas faixas de composição. Outra característica que pode ser ressaltada é o também extenso campo de fase tetragonal. Isto permite a preparação de cerâmicas de zircônia tetragonal policristalina dopada com cálcia, de forma análoga aos dopantes $\mathrm{Y}_{2} \mathrm{O}_{3}$ e $\mathrm{CeO}_{2}$. Assim, dependendo do teor de $\mathrm{CaO}$ e das condições de processamento e dos tratamentos térmicos (sinterização e envelhecimento) realizados, é possível obter a zircônia parcialmente estabilizada (CaPSZ) ou totalmente estabilizada na fase cúbica (Ca-FSZ), ou ainda a chamada zircônia tetragonal policristalina contendo cálcia (Ca-TZP) [14].

A zircônia totalmente estabilizada com cálcia (CaFSZ) tem sido muito estudada porque é a que apresenta maior condutividade iônica para este tipo de estabilizante. Analogamente ao que ocorre para outras cerâmicas de zircônia dopada, a condutividade iônica apresenta um valor máximo dependente do teor de estabilizante, em geral, para o teor mínimo necessário para a total estabilização da fase cúbica. Para a zircônia-cálcia este teor está entre 12 e 13\% mol de $\mathrm{CaO}$. Por outro lado, a zircônia tetragonal policristalina contendo cálcia (Ca-TZP) foi muito pouco estudada até o momento. Isto está provavelmente relacionado com a dificuldade de sua obtenção, uma vez que requer o emprego de pós ultrafinos e que a retenção da estrutura tetragonal só foi obtida com o uso da técnica de sinterização rápida [14].

A estabilização das diversas estruturas cristalográficas na zircônia é influenciada pelas dimensões das partículas e dos grãos, em materiais particulados e em cerâmicas sinterizadas, respectivamente. A estrutura de alta temperatura, e também de alta simetria é, em geral, preferencialmente obtida em materiais com dimensões pequenas, e esta dependência é comumente descrita por meio de modelos termodinâmicos derivados da equação de Gibbs-Thompson, considerando o tamanho de partículas (ou grãos) e os efeitos de superfície.

O tamanho mínimo de grãos necessário para reter a estrutura tetragonal na zircônia, denominado tamanho crítico, depende do tipo e do teor do dopante. Assim, em cerâmicas sinterizadas de zircônia contendo 3\% mol ítria, o tamanho crítico para a retenção da fase tetragonal é de 250 nm, enquanto que para a zircônia com 4\% mol cálcia, o tamanho crítico é $150 \mathrm{~nm}[15,16]$. Desta forma, a retenção da fase tetragonal metaestável não está somente relacionada com a obtenção de pós nanométricos mas também, com as etapas de processamento subseqüentes, que devem ser adequadas para simultaneamente produzir uma cerâmica densa e contendo grãos menores que o tamanho crítico.

A zircônia parcialmente estabilizada com cálcia, em geral consiste de uma mistura de fases tetragonal e cúbica ou monoclínica e cúbica. Este material foi particularmente estudado por suas propriedades mecânicas, especialmente sua alta resistência ao choque térmico [17]. Na faixa de composições que compreende a zircônia parcialmente estabilizada, os pós comerciais e os tratamentos térmicos convencionais não permitem a obtenção da mistura de fases cúbica mais tetragonal, c $+\mathrm{t}$. De forma geral, durante o resfriamento da cerâmica os grãos com estrutura tetragonal crescem ultrapassando o tamanho crítico, e espontaneamente se transformam formando a fase monoclínica, resultando numa composição de fases cúbica mais monoclínica, c + m.

A resistividade iônica de cerâmicas de zircônia dopada depende do tamanho médio de grãos, da composição química e do teor de fases, além dos tratamentos térmicos. A pureza dos materiais de partida também é importante porque em temperaturas intermediárias, as impurezas segregadas nos contornos de grão da cerâmica sinterizada, em geral, causam um bloqueio adicional aos portadores de carga nessas regiões.

Foi mostrado anteriormente [18], em cerâmicas de zircônia parcialmente estabilizadas com magnésia, que o efeito da fase monoclínica é semelhante ao de impurezas e outros defeitos (poros e fases secundárias) localizados nos contornos de grão, isto é, a fase monoclínica exerce um bloqueio extra aos portadores de carga. Esta hipótese pode ser feita levando em conta que as diversas fases na zircônia possuem resistividades diferentes. Além disso, a fase monoclínica é a que apresenta maior resistividade e, esta diferença, em resistividade a uma dada temperatura, é tal que a fase monoclínica pode ser considerada uma fase isolante. Isto não acontece com as fases cúbica e tetragonal, que apresentam resistividades muito próximas na região de temperaturas entre 200 e $600{ }^{\circ} \mathrm{C}$, como demonstrado em amostras mono- e policristalinas [19, 20].

Apesar do número de trabalhos realizados com este tipo de cerâmica ser consideravelmente grande, boa parte destes dedicou atenção especial à evolução da microestrutura ou às propriedades mecânicas das cerâmicas sinterizadas, ou ainda à síntese de pós ultrafinos, visando a obtenção de propriedades otimizadas. Relativamente poucos trabalhos foram feitos focalizando o estudo do comportamento elétrico com a evolução de fases nas zircônias dopadas, especialmente na zircônia-cálcia. Assim, neste trabalho, cerâmicas sinterizadas de zircônia-11,5\% mol cálcia foram preparadas a partir de pós nanométricos obtidos por coprecipitação. A sinterização dos compactos foi feita a uma mesma temperatura e com diferentes tempos, para verificar o efeito da composição de fases na resistividade elétrica da cerâmica sinterizada.

\section{EXPERIMENTAL}

$\mathrm{ZrOCl}_{2} \cdot 8 \mathrm{H}_{2} \mathrm{O}(>98 \%, \mathrm{BDH})$ e $\mathrm{CaCO}_{3}(99 \%$, B. Herzog) foram utilizados como materiais de partida. Os demais reagentes utilizados são de grau analítico. A 
técnica de preparação da solução sólida utilizada foi a coprecipitação dos hidróxidos. A solução inicial dos cátions foi preparada em água deionizada, na proporção desejada. Após homogeneização da solução contendo a mistura dos cátions de interesse, foi adicionada uma solução aquosa contendo o agente precipitante $\left(\mathrm{NH}_{4} \mathrm{OH}\right)$. O precipitado formado foi lavado e coletado a vácuo. A seqüência de lavagens consistiu de uma solução diluída de amônia, seguida de etanol e isopropanol. A última etapa de desidratação do precipitado foi realizada com a destilação azeotrópica em álcool $n$ butílico. Detalhes sobre os parâmetros utilizados na síntese podem ser encontrados em [21].

O gel seco foi calcinado a uma temperatura determinada a partir dos resultados de análise térmica. Amostras cilíndricas foram preparadas por compactação uniaxial a $98 \mathrm{MPa}$ em matriz de aço inox. A sinterização dos compactos foi realizada ao ar em forno tubular à temperatura de $1500{ }^{\circ} \mathrm{C}$ com tempos de permanência nesta temperatura de $2 \mathrm{~h}$ ou $3 \mathrm{~min}$. Durante o processamento dos pós não foram utilizados quaisquer aditivos, e não foram realizadas etapas de moagem, classificação ou peletização, que poderiam ocasionar a contaminação do material calcinado.

A decomposição térmica do gel seco foi estudada por análise termogravimétrica (TG) e térmica diferencial (DTA) simultânea (STA409, Netzsch), da temperatura ambiente até $1200^{\circ} \mathrm{C}$ com taxa de aquecimento de $10^{\circ} \mathrm{C} \cdot \mathrm{min}^{-1}$, em atmosfera dinâmica de ar sintético $\left(\sim 20 \% \mathrm{O}_{2}\right)$, e utilizando alumina como material de referência. $\mathrm{O}$ teor de cálcio foi determinado por fluorescência de raios X (Shimadzu EDX-700) em amostras calcinadas. $\mathrm{O}$ valor da área de superfície específica, $\mathrm{S}$, foi determinado pelo método BET (Brunauer, Emmett e Teller), pela técnica de adsorção gasosa (ASAP 2010, Micromeritics), após desgaseificação a $200{ }^{\circ} \mathrm{C}$. A densificação de compactos foi estudada pela retração linear (DIL 402E/7, Netzsch) com taxa de aquecimento de $8{ }^{\circ} \mathrm{C}$. min $^{-1}$ até $1650^{\circ} \mathrm{C}$.

Espectros Raman foram obtidos em amostra sinterizadas utilizando um espectrômetro Renishaw Raman Microscope 3000, acoplado a um microscópio Olympus BH-2 e a um detector CCD. Foi utilizada a radiação excitante em 632,8 nm de um laser de He-Ne (Spectra Physics, modelo 127). A superfície de fratura de amostras sinterizadas foi observada em microscópios eletrônicos de varredura (LEO 440i, Oxford e XL30, Philips) por elétrons secundários. Medidas de resistividade elétrica foram feitas por espectroscopia de impedância (4192A, Hewlett Packard) na faixa de frequiência de $5 \mathrm{~Hz}$ a $13 \mathrm{MHz}$, com amplitude de sinal igual a $50 \mathrm{mV}$. Para estas medidas, foram utilizados eletrodos de prata, aplicados por pintura e queima a $500^{\circ} \mathrm{C}$. Os resultados apresentados incluem a normalização para as dimensões das amostras analisadas, mas não foram corrigidos com relação à porosidade.

\section{RESULTADOS E DISCUSSÃO}

A Fig. 1 mostra as curvas termogravimétrica e sua derivada obtidas para o gel seco. A perda total de massa até $1200{ }^{\circ} \mathrm{C}$ é de $20 \%$, sendo que a maior perda de massa ocorre entre 100 e $500{ }^{\circ} \mathrm{C}$. A derivada da curva termogravimétrica indica duas regiões de perda de massa, a primeira em temperaturas inferiores a $250{ }^{\circ} \mathrm{C}$, está relacionada com a eliminação de água fisicamente adsorvida e parte dos resíduos de álcool. Sabendo que a eliminação dos grupos butóxido, decorrentes da destilação com $n$-butanol, ocorre a temperaturas superiores a $250{ }^{\circ} \mathrm{C}$ [22], a perda de massa em temperaturas entre 250 e $400{ }^{\circ} \mathrm{C}$, é atribuída à evaporação de resíduos de álcool provenientes da etapa de desidratação do gel.

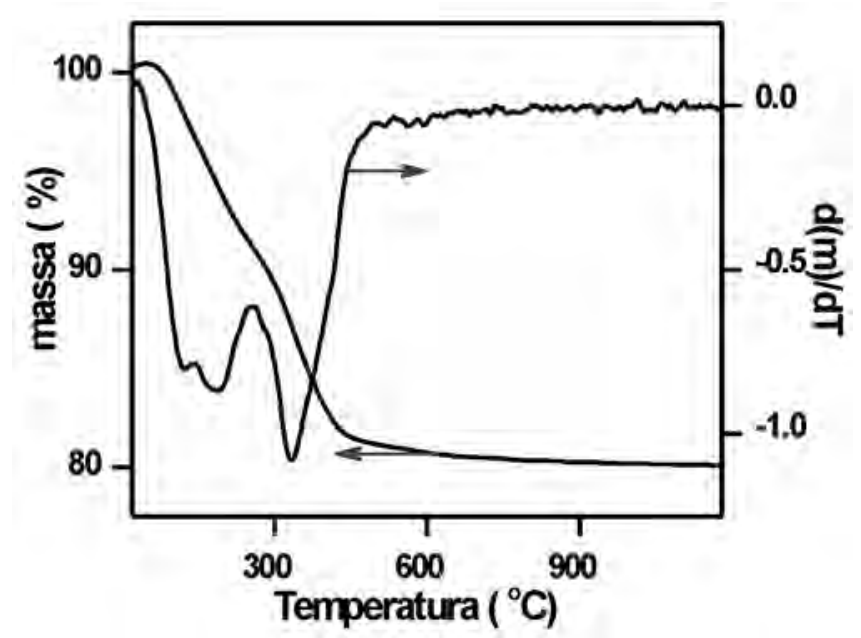

Figura 1: Curvas termogravimétrica e derivada do gel seco.

[Figure 1: Thermogravimetry and differential thermogravimetry curves of the dried gel.]

A curva de análise térmica diferencial do gel seco é mostrada na Fig. 2. Esta curva apresenta eventos exotérmicos devidos à eliminação de resíduos dos diversos tipos de álcool utilizados na lavagem e desidratação do gel, além

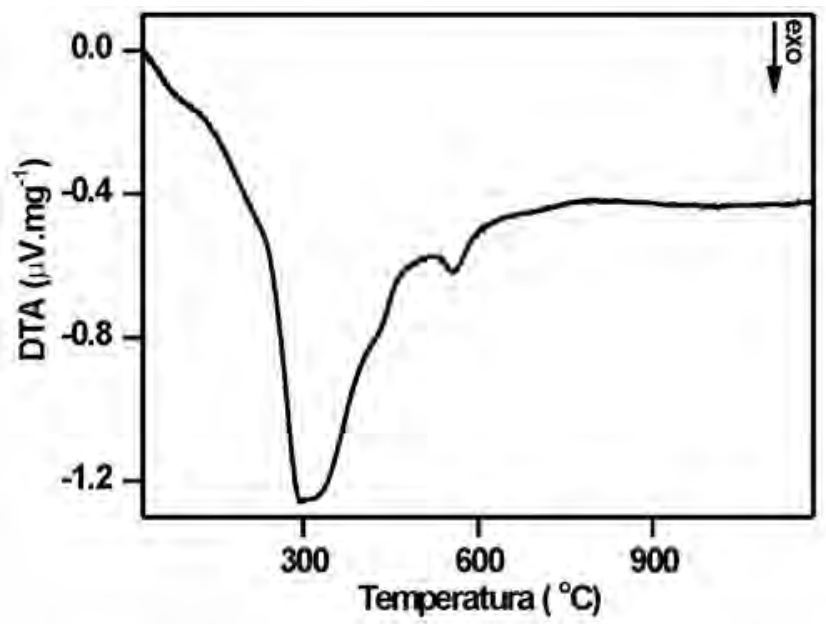

Figura 2: Curva de análise térmica diferencial do gel seco. [Figure 2: Differential thermal analysis curve of the dried gel.] 
de um pico exotérmico a $570{ }^{\circ} \mathrm{C}$, que não é acompanhado de perda de massa (Fig. 1), e que pode ser associado com a cristalização do material. A partir destes resultados foi escolhida a temperatura de $550{ }^{\circ} \mathrm{C}$ para a calcinação dos géis. $\mathrm{O}$ tempo de permanência nesta temperatura foi de $2 \mathrm{~h}$.

O teor do aditivo determinado, após a calcinação do precipitado e assumindo que todo o cálcio está na forma de $\mathrm{CaO}$, foi de $11,5 \%$ mol. Para este teor de aditivo, pode-se dizer que a solução sólida obtida deverá dar origem a um material sinterizado consistindo de zircônia parcialmente estabilizada com cálcia, de acordo com o diagrama de fases [13]. Além disso, é também esperado que amostras sinterizadas apresentem uma composição de fases cúbica (majoritária) e tetragonal ou monoclínica (minoritária).

$\mathrm{O}$ valor da área de superfície específica, $\mathrm{S}$, obtida pelo método de BET na amostra calcinada, foi de $136 \mathrm{~m}^{2} \cdot \mathrm{g}^{-1}$, mostrando que a técnica utilizada permitiu a obtenção de pós ultrafinos de zircônia-cálcia.

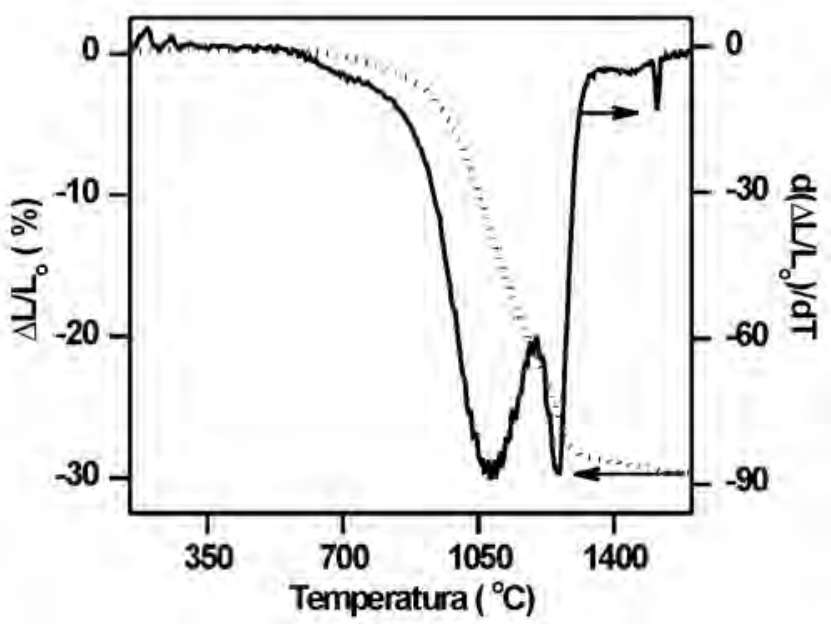

Figura 3: Curva de retração linear do compacto.

[Figure 3: Linear shrinkage curve of a powder compact.]

A Fig. 3 apresenta a curva de retração linear obtida para um compacto preparado com a zircônia-cálcia calcinada. A variação dimensional até a temperatura de $800{ }^{\circ} \mathrm{C}$ é desprezível. A maior parte da retração linear ocorre entre $800{ }^{\circ} \mathrm{C}$ e $1500{ }^{\circ} \mathrm{C}$, sendo a taxa de retração mais acentuada entre $1000{ }^{\circ} \mathrm{C}$ e $1200{ }^{\circ} \mathrm{C}$. A retração total do compacto foi de $30 \%$ e, portanto, semelhante à de outras cerâmicas à base de zircônia preparadas por esta técnica de síntese [21]. Apesar da similaridade no valor da retração total, as curvas de retração linear das zircônias tetragonais policristalinas apresentam um comportamento único, ou seja, a derivada da curva de retração apresenta uma única inflexão, ao contrário do que ocorre para as zircônias parcialmente e totalmente estabilizadas na fase cúbica. Esta diferença pode ser entendida como o resultado do crescimento de grãos que é dependente do teor de dopante. É sabido da literatura [23] que grãos com estrutura cúbica apresentam tamanho comparativamente grande ( $>1 \mu \mathrm{m})$ e porosidade interna, enquanto que grãos com estrutura monoclínica ou tetragonal têm tamanho médio relativamente pequeno e apresentam porosidade intragranular desprezível. Isto é o resultado de diferenças nas taxas de sinterização das diferentes fases da zircônia. Em geral, pós de zircônia contendo aditivos preparados por técnicas de solução, contêm partículas muito finas que promovem a estabilização da fase tetragonal após a calcinação. Então, compactos preparados com esses pós densificam rapidamente (alta taxa de retração) a baixas temperaturas, mas para altas temperaturas, especialmente quando a temperatura no compacto corresponde à dos campos de fase c ou $t+c$, a taxa de sinterização diminui e a densificação é lenta, prevalescendo o crescimento dos grãos. A curva de retração linear da Fig. 3 comprova essa diferença na taxa de retração na zircônia parcialmente estabilizada com cálcia.
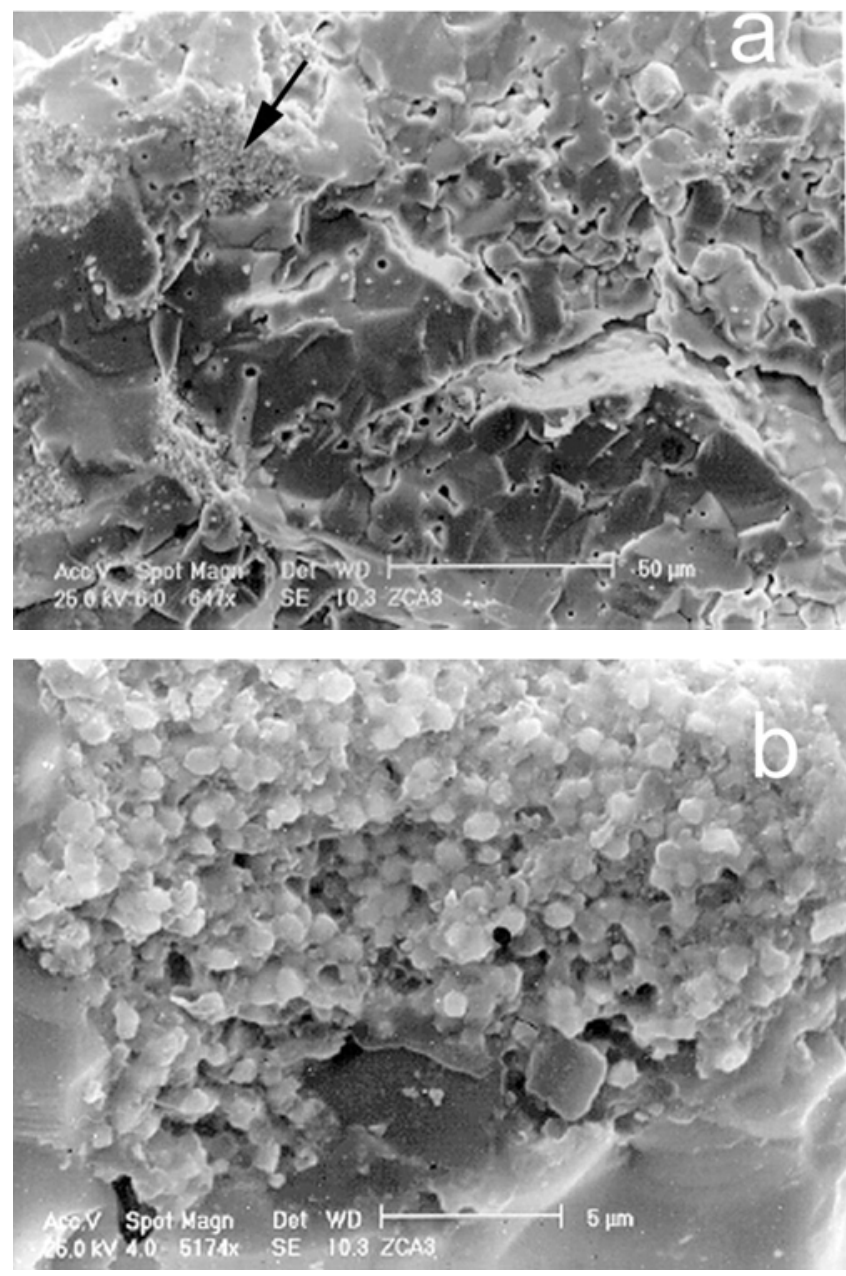

Figura 4: a) Micrografia obtida em microscópio eletrônico de varredura da superfície de fratura de amostra sinterizada com patamar de 2 h; b) micro-região assinalada em (a) com maior aumento.

[Figure 4: Scanning electron microscopy micrograph of a fractured surface of the sintered pellet with 2 h soaking time; b) high magnification of the region shown by arrow in (a).] 
Uma micrografia representativa da superfície de fratura de uma cerâmica sinterizada com tempo de patamar de 2 h, obtida por microscopia eletrônica de varredura, é mostrada na Fig. 4a. O modo de fratura é misto, isto é, parte transgranular e parte intergranular. A fratura transgranular ocorre nos grãos maiores (cúbicos), enquanto que a fratura intergranular é observada nos grãos menores (monoclínicos ou tetragonais). Para esta composição é esperada que a maior parte da cerâmica sinterizada apresente estrutura cúbica e, portanto, grãos relativamente grandes contendo porosidade fechada. A fase minoritária tetragonal ou monoclínica, em geral é observada nos contornos de grão, neste caso, distribuída de forma irregular. A região assinalada na Fig. 4a é mostrada com maior aumento na Fig. 4b. O tamanho médio estimado desses grãos menores é de $200 \mathrm{~nm}$.

O espectro Raman da cerâmica sinterizada a $1500{ }^{\circ} \mathrm{C}$ por 2 h é mostrado na Fig. 5. Diversas bandas Raman são observadas. Os modos ativos Raman para as diferentes estruturas cristalográficas da zircônia já foram determinados por análise de grupo-fator [24]. Assim, é esperado que a fase monoclínica (grupo espacial P2/c) apresente 18, a fase tetragonal (grupo espacial P4 $/ \mathrm{nmc}$ ) 6, e a fase cúbica (grupo espacial Fm3m) 1 modo ativo Raman. Dessa forma, o espectro da Fig. 5 corresponde ao de uma zircônia parcialmente estabilizada contendo as fases monoclínica e cúbica.

A microestrutura da cerâmica sinterizada a $1500{ }^{\circ} \mathrm{C}$ por 3 min é mostrada na Fig. 6a. Pode-se observar que o modo de fratura é também misto, mas que ao contrário da amostra sinterizada por $2 \mathrm{~h}$, o modo intergranular de fratura é predominante. São também observados poros intra- e intergranulares com tamanhos diferentes. Da mesma forma que para a amostra sinterizada por $2 \mathrm{~h}$, diversas micro-regiões contendo grãos pequenos são observadas e sua distribuição é irregular no compacto. Uma destas micro-regiões é mostrada com maior aumento na Fig. 6b.

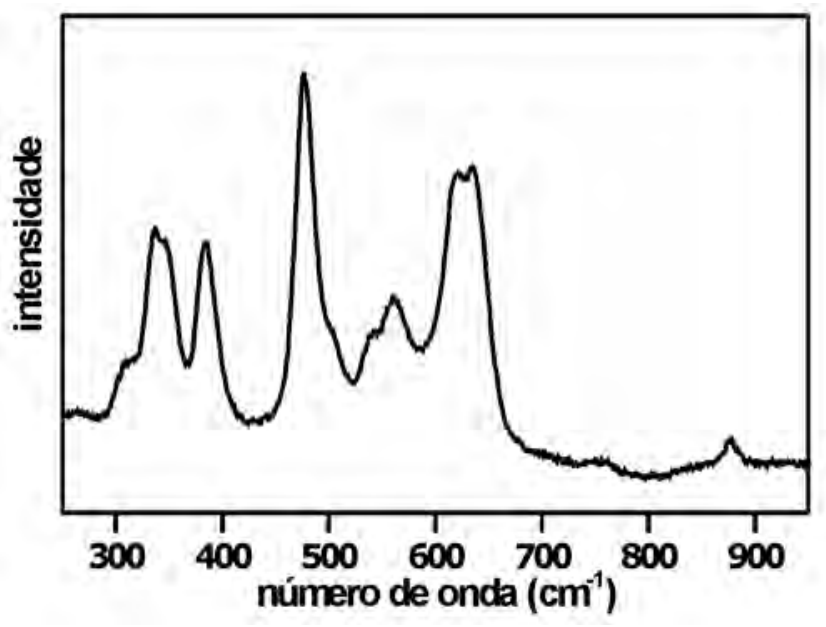

Figura 5: Espectro Raman da amostra sinterizada com patamar de $2 \mathrm{~h}$ a $1500^{\circ} \mathrm{C}$.

[Figure 5: Raman spectrum of the specimen sintered at $1500{ }^{\circ} \mathrm{C}$ for $2 h$.
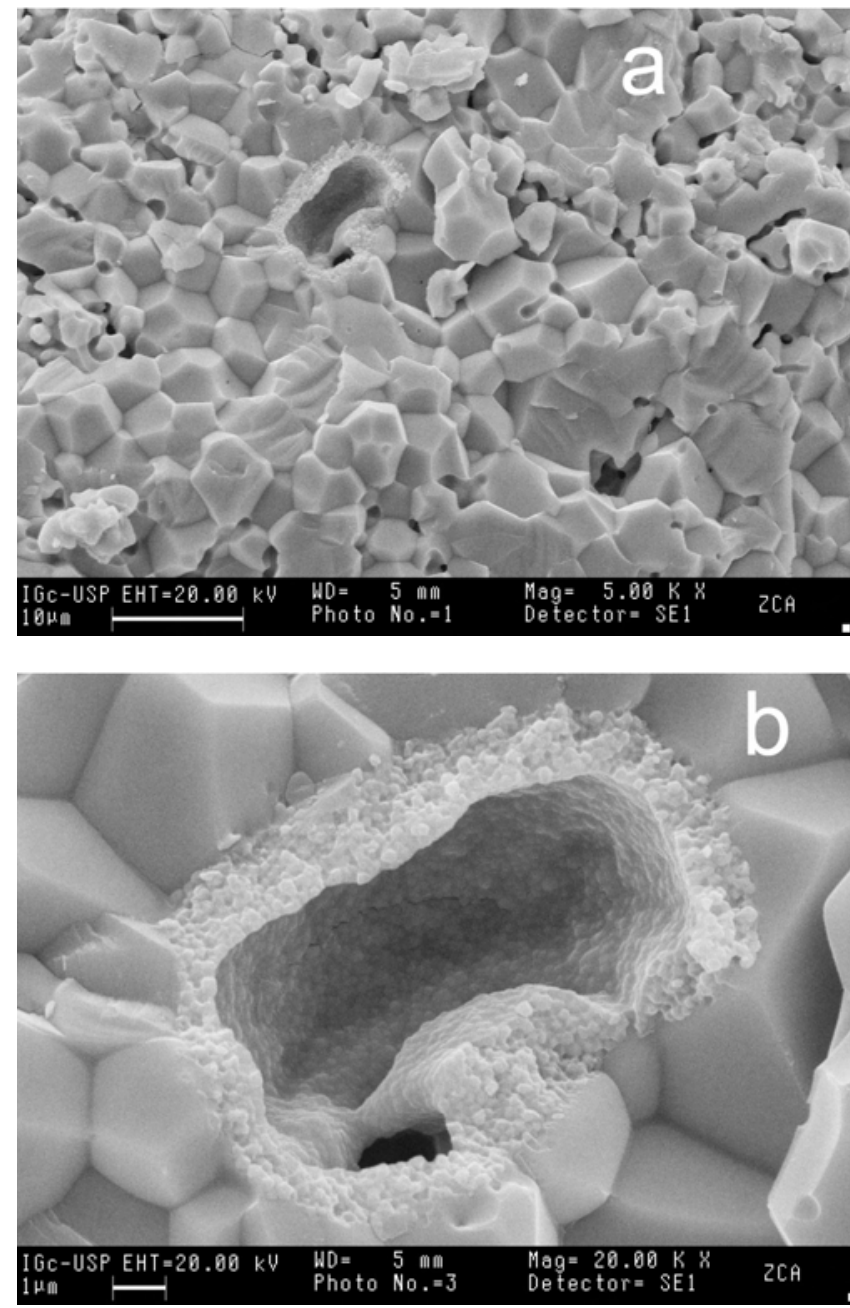

Figura 6: a) Micrografia obtida em microscópio eletrônico de varredura da superfície de fratura de amostra sinterizada com patamar de 3 min; b) micro-região com maior aumento.

[Figure 6: Scanning electron microscopy micrograph of a fractured surface of the sintered pellet with 3 min. soaking time; b) high magnification of a micro-region.]

Esses grãos de tamanho pequeno podem estar relacionados com as fases tetragonal e/ou monoclínica, como mencionado anteriormente.

O espectro Raman da amostra sinterizada por 3 min a $1500{ }^{\circ} \mathrm{C}$ é mostrado na Fig. 7. Uma única banda alargada próxima a $600 \mathrm{~cm}^{-1}$ e característica da fase cúbica, é detectada. Não são observadas bandas relacionadas com a fase monoclínica e nem com a fase tetragonal. Entretanto, pela microestrutura mostrada na Fig. 6, é esperado que esta amostra apresente uma mistura de fases c + t ou c + m. Uma explicação para isto está na concentração muito pequena da fase tetragonal ou monoclínica em comparação com a fase cúbica majoritária. Além disso, para a observação no microscópio eletrônico de varredura, somente a superfície de fratura foi utilizada, enquanto que para a espectroscopia Raman, a superfície de observação e análise foi a face maior das amostras. A observação prévia dessa superfície 
no microscópio óptico acoplado ao espectrômetro Raman, não revelou a existência de grãos com tamanhos pequenos, indicando que os grãos com morfologia mostrada nas Figs. 6a e 6b estão concentrados no interior do compacto.

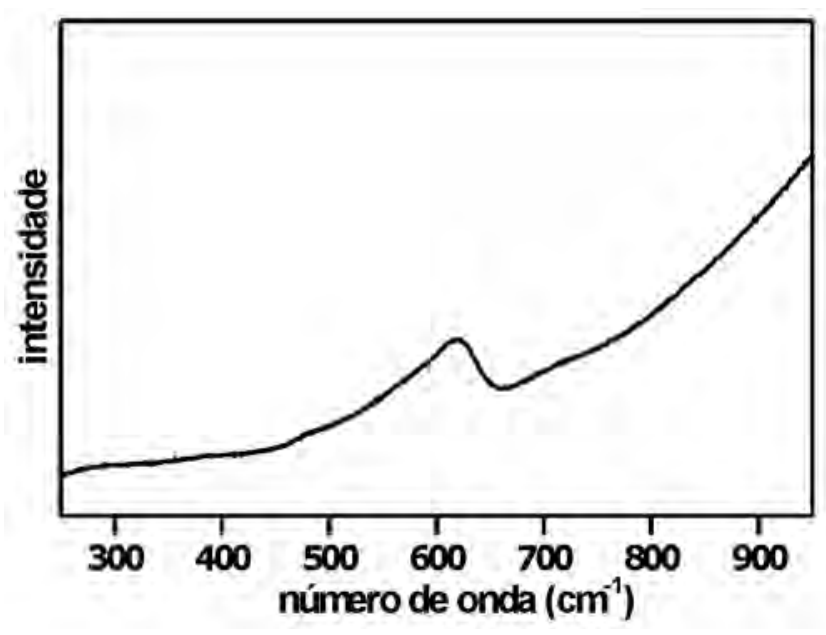

Figura 7: Espectro Raman da amostra sinterizada com patamar de 3 min a $1500{ }^{\circ} \mathrm{C}$.

[Figure 7: Raman spectrum of the specimen sintered at $1500{ }^{\circ} \mathrm{C}$ for $3 \mathrm{~min}$.

A Fig. 8 mostra diagramas de impedância obtidos a $494{ }^{\circ} \mathrm{C}$ para as amostras sinterizadas por diferentes tempos a $1500{ }^{\circ} \mathrm{C}$. Os números nestes diagramas representam o logaritmo decimal da freqüência (em Hz). Em toda a faixa de temperaturas de medidas, as amostras analisadas

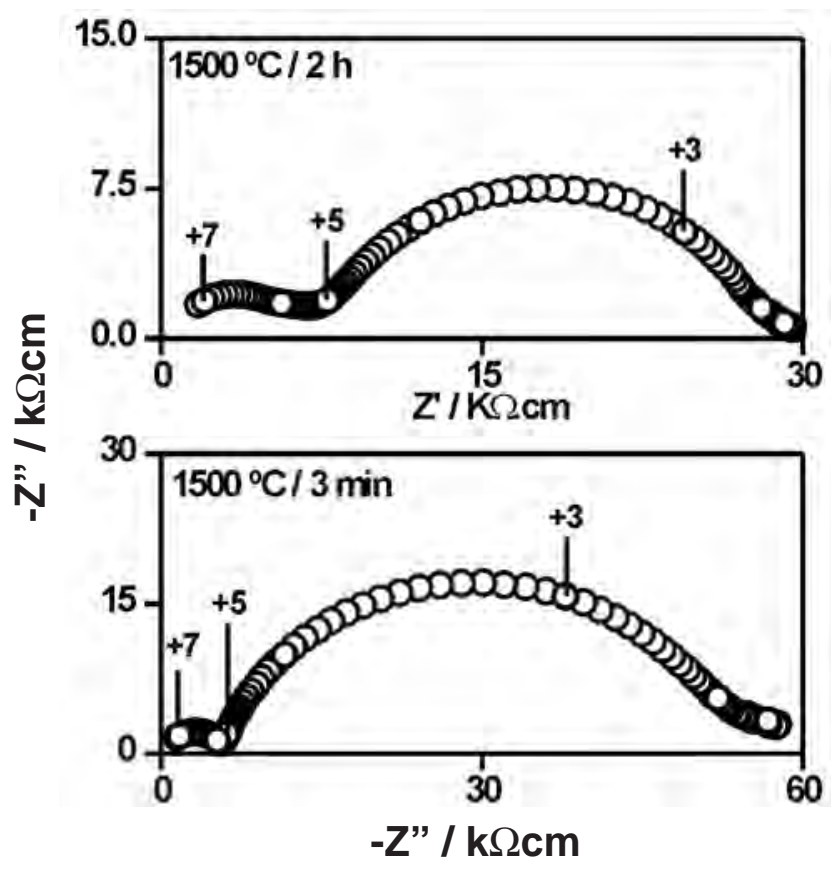

Figura 8: Diagramas de impedância de amostras sinterizadas. Temperatura de medida $=495^{\circ} \mathrm{C}$.

[Figure 8: Impedance spectroscopy diagrams of sintered specimens. Measuring temperature $\left.=495^{\circ} \mathrm{C}\right]$ apresentam um semicírculo de alta freqüência, atribuído aos efeitos capacitivos e resistivos dos grãos e um semicírculo de baixa freqüência devido ao bloqueio dos portadores de carga nas interfaces dos grãos. A partir dos diâmetros destes semicírculos foram calculadas as resistividades intragranular e intergranular, respectivamente.

A amostra sinterizada por $2 \mathrm{~h}$ apresenta ainda um semicírculo em freqüência intermediária. Semicírculos na faixa intermediária de freqüência são normalmente atribuídos a porosidade, trincas e microtrincas ou fases com diferenças substanciais em resistividade [18, 25-27]. A porosidade aumenta a resistividade e isto é observado de duas formas nos diagramas de impedância: por meio de aumento no diâmetro do semicírculo correspondente, ou como um semicírculo em freqüência intermediária. O primeiro tipo de bloqueio foi observado preferencialmente em relação à porosidade intragranular [28], enquanto que o segundo foi relacionado com a porosidade intergranular [25]. Assim, a cerâmica sinterizada por 2 h e contendo porosidade intragranular, deve apresentar maior resistividade elétrica comparada à de uma amostra com igual composição, mas que não apresente porosidade no interior dos grãos sem, entretanto, dar origem a um semicírculo adicional no diagrama de impedância. Trincas e microtrincas também podem gerar um bloqueio aos portadores de carga. Entretanto, se este efeito fosse o responsável pelo semicírculo de faixa intermediária de freqüência, seria esperado que ocorresse também na amostra aquecida e resfriada mais rapidamente no processo de sinterização. Assim, é mais razoável associar este semicírculo à resistividade da fase monoclínica, evidenciada no espectro Raman desta amostra (Fig. 5).

É importante notar que a amostra sinterizada por 3 min não apresenta outro semicírculo na região intermediária em freqüência apesar de não possuir fase única. Este resultado mostra que os grãos de tamanho relativamente pequeno,

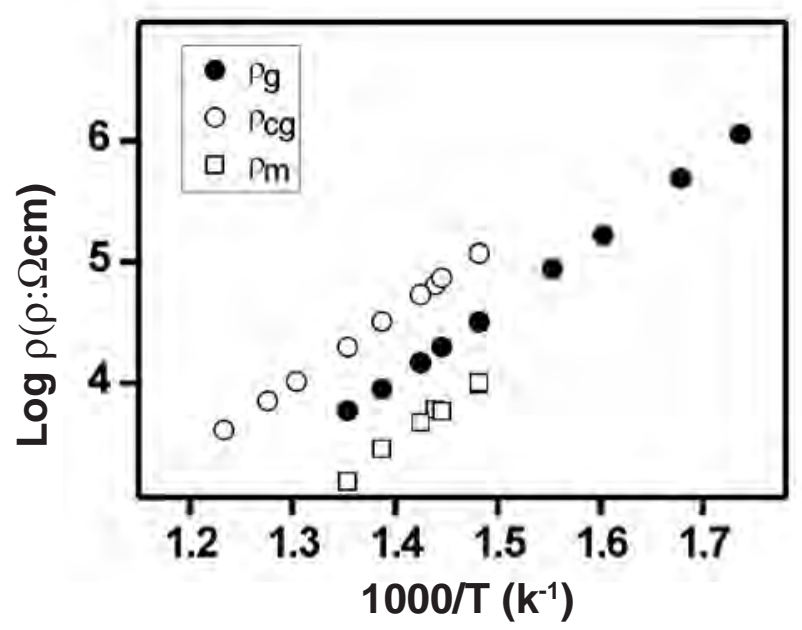

Figura 9: Gráficos de Arrhenius da resistividade elétrica da amostra sinterizada a $1500{ }^{\circ} \mathrm{C}$ por $2 \mathrm{~h}$

[Figure 9: Arrhenius plots of specimens sintered at $1500{ }^{\circ} \mathrm{C}$ for $2 \mathrm{~h}$. ] 
observados nas micrografias mostradas na Fig. 6, devem estar relacionados com a fase tetragonal minoritária. Neste caso, a resistividade intragranular representa a soma das contribuições devidas aos grãos com simetria cúbica e tetragonal, uma vez que estes apresentam resistividades muito próximas na região de temperaturas de medidas, como mencionado anteriormente.

Os gráficos de Arrhenius das resistividades intragranular, intergranular e devida à fase monoclínica da amostra sinterizada por 2 h são mostrados na Fig. 9. Na faixa restrita de temperaturas de medida é linear a dependência das resistividades com o inverso da temperatura absoluta, e não é observada mudança de inclinação. Além disso, os segmentos de reta são aproximadamente paralelos, mostrando que o mecanismo de condução é o mesmo. Como esperado, apesar da fase monoclínica se comportar como um elemento bloqueante à migração dos portadores de carga, sua contribuição para a resistividade total é pequena, em conseqüência da fração relativa de fase monoclínica na matriz cúbica.

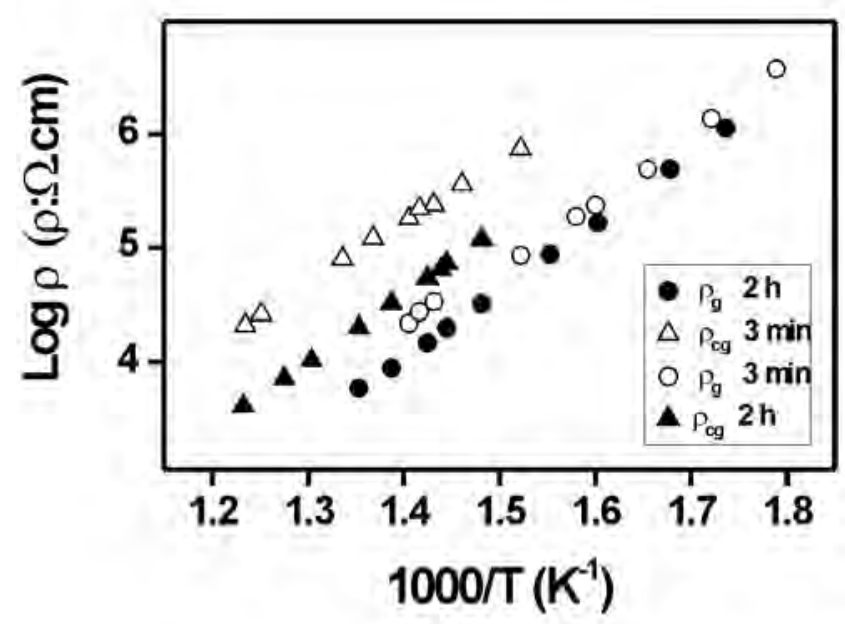

Figura 10: Gráfico de Arrhenius dos componentes intra e intergranular das amostras sinterizadas.

[Figure 10: Arrhenius plots of intra and intergranular components of the resistivity for sintered pellets.]

A amostra sinterizada por $3 \mathrm{~min}$ apresenta comportamento similar ao mostrado na Fig. 9 para os componentes intra e intergranular da resistividade. Na Fig. 10 são mostrados os gráficos de Arrhenius das resistividades intragranular e intergranular das duas amostras, para fins comparativos. Como a resistividade intragranular se deve somente aos grãos da fase cúbica na amostra sinterizada por $2 \mathrm{~h}$, esta deve apresentar resistividade pouco inferior à da amostra sinterizada por $3 \mathrm{~min}$, na qual este parâmetro representa a soma das resistividades dos grãos cúbicos e tetragonais. Os resultados mostrados na Fig. 10 corroboram esta hipótese. Com relação ao componente intergranular da resistividade, a amostra sinterizada por $3 \mathrm{~min}$ apresenta maior resistividade por possuir grãos com tamanho inferior ao da amostra sinterizada por 2 h. Em termos da contribuição da fase intergranular para a resistividade, este efeito é descrito por um maior número de contornos de grão por unidade de área.

A partir dos gráficos de Arrhenius da resistividade elétrica das amostras de $\mathrm{ZrO}_{2}: 11,5 \% \mathrm{~mol} \mathrm{CaO}$ foram calculados os valores de energia de ativação aparente do processo de condução (Tabela I). Para todos os componentes da resistividade elétrica nessas amostras os valores obtidos estão entre 1,05 e 1,20 eV, de acordo com o esperado para condutores de íons oxigênio. Pode também ser observado nesta Tabela que os valores de energia aparente de ativação para a amostra sinterizada por 3 min são um pouco inferiores aos da amostra sinterizada com patamar de $2 \mathrm{~h}$. Considerando que em amostras de zircônia-ítria e zircônia-céria a energia de ativação para a migração de portadores de carga na fase tetragonal é um pouco inferior ao da fase cúbica, esta pequena diferença é uma indicação adicional que os grãos de menor tamanho nesta amostra apresentam simetria tetragonal. Os valores da condutividade total das amostras a $400{ }^{\circ} \mathrm{C}$ é também mostrado na Tabela I. O valor obtido para a amostra sinterizada a $1500{ }^{\circ} \mathrm{C}$ por 2 h é próximo aos valores mais elevados obtidos para cerâmicas de $\mathrm{ZrO}_{2}$ : $\mathrm{CaO}$ totalmente estabilizadas $\left(\sigma=7,2 \times 10^{-6} \mathrm{~S} \mathrm{~cm}^{-1}\right.$ para composições de 12 a $13 \%$ mol CaO [29]).

Os resultados de resistividade elétrica nas cerâmicas de zircônia parcialmente estabilizada com cálcia sinterizadas por diferentes tempos permitiram evidenciar as contribuições relativas das diferentes fases cristalinas na resistividade elétrica total deste eletrólito sólido.

Tabela I - Valores de energia aparente de ativação dos componentes intragranular $\left(E_{g}\right)$, intergranular $\left(E_{c g}\right)$ e fase monoclínica $\left(\mathrm{E}_{\mathrm{m}}\right)$ da resistividade elétrica de cerâmicas de $\mathrm{ZrO}_{2}: 10,5 \%$ mol $\mathrm{CaO}$. Valores calculados para a condutividade elétrica total $\left(\sigma_{t}^{400}\right)$ a $400{ }^{\circ} \mathrm{C}$.

[Table I-Apparent activation energy values for intragranular $\left(E_{g}\right)$, intergranular $\left(E_{c g}\right)$ and monoclinic phase $\left(E_{m}\right)$ of the electrical resistivity in $\mathrm{ZrO}_{2}: 12.5 \mathrm{~mol} \% \mathrm{CaO}$. Calculated values of total electrical conductivity $\left(\sigma_{t}^{400}\right)$ at $400{ }^{\circ} \mathrm{C}$.]

\begin{tabular}{ccc}
\hline & \multicolumn{2}{c}{$\mathrm{ZrO}_{2}: 11,5 \% \mathrm{~mol} \mathrm{CaO}$} \\
& $1500^{\circ} \mathrm{C} / 2 \mathrm{~h}$ & $1500{ }^{\circ} \mathrm{C} / 3 \mathrm{~min}$ \\
\hline $\mathbf{E}_{\mathbf{g}}(\mathbf{e V})$ & 1,19 & 1,12 \\
$\mathbf{E}_{\mathrm{cg}}(\mathbf{e V})$ & 1,17 & 1,06 \\
$\mathbf{E}_{\mathbf{m}}(\mathbf{e V})$ & 1,21 & -- \\
$\sigma_{\mathbf{t}}^{400}\left(\mathbf{S} . \mathbf{c m}^{-1}\right)$ & $6,1 \times 10^{-6}$ & $2,4 \times 10^{-6}$ \\
\hline
\end{tabular}

\section{CONCLUSÕES}

Foi obtida a solução sólida $\mathrm{ZrO}_{2}: 11,5 \%$ mol CaO pela técnica da co-precipitação. A decomposição completa do gel precursor foi obtida a uma temperatura relativamente 
baixa, resultando num material com alta área de superfície específica. A taxa de retração de compactos preparados com esse material é máxima entre 1000 e $1200{ }^{\circ} \mathrm{C}$, diminuindo a temperaturas superiores por causa de mudança de fase cristalina na zircônia. Amostras sinterizadas por $2 \mathrm{~h} \mathrm{a}$ $1500{ }^{\circ} \mathrm{C}$ apresentam um espectro Raman característico da mistura de fases cúbica e monoclínica. No espectro Raman de amostras sinterizadas por $3 \mathrm{~min}$ só foi identificado o modo vibracional característico da fase cúbica. Observações da microestrutura das amostras sinterizadas por diferentes tempos de patamar revelou grãos com tamanhos distintos, distribuídos irregularmente. Medidas de espectroscopia de impedância confirmaram a presença da fase monoclínica em amostras sinterizadas por $2 \mathrm{~h}$ a $1500^{\circ} \mathrm{C}$. As amostras de zircônia parcialmente estabilizada com cálcia, sinterizadas com patamar de 3 min, apresentam as fases tetragonal e cúbica cujas contribuições para a resistividade total do eletrólito não são separáveis no domínio de freqüência.

\section{AGRADECIMENTOS}

A FAPESP (97/14238-4, 94/05929-5, 95/05172-4 e 95/05635-4), CNPq e CNEN pelo suporte financeiro. Aos laboratórios de Cerâmicas Estruturais do IPEN pela análise de retração linear, e de Espectroscopia Molecular do Instituto de Química da USP pelas medidas de espectroscopia Raman. R. C. Buissa Neto agradece à FAPESP pela bolsa de estudos.

\section{REFERÊNCIAS}

[1] R. C. Garvie, in High Temperature Oxides - Part II, ed. A. M. Alper, Academic Press, New York 1(970) p. 117-165. [2] E. C. Subbarao, in Advances in Ceramics v. 3, Science and Tecnology of Zirconia I, ed. A. H. Heuer, L. W. Hobbs, Am. Ceram. Soc., Columbus, Ohio (1981) p. 1-24.

[3] T. Y. Tien, E. C. Subbarao, J. Chem. Phys. 39 (1963) 1041.

[4] T. Y. Tien, J. Appl. Phys. 35 (1964) 122.

[5] D. J. Green, D. R. Maki, P. S. Nicholson, J. Am. Ceram. Soc. 57 (1974) 136.

[6] N. M. Beekmans, L. Heyne, Electrochim. Acta 21 (1976) 303.

[7] S. H. Chu, M. A. Seitz, J. Solid State Chem. 23 (1978) 297.

[8] R. M. Dickerson, M. V. Swain, A. H. Heuer, J. Am.
Ceram. Soc. 70 (1987) 214.

[9] M. Aoki, Y.-M. Chiang, I. Kosacki, L. J.-R. Lee, H. L. Tuller, Y. Lin, J. Am. Ceram. Soc. 79 (1996) 1169.

[10] B. C. H. Steele, J. Drennan, R. K. Slotwinski, N. Bonanos, E. P. Butler, Advances in Ceramics v. 3, Science and Tecnology of Zirconia I, ed. A. H. Heuer, L. W. Hobbs, Am. Ceram. Soc., Columbus, Ohio (1981) p. 286.

[11] R. Stevens, Magnesium Elektron Publication $n^{\circ}$ 113, 1 (986).

[12] I. Birky, R. Stevens, Key Eng. Mater. 122-124 (1996) 527.

[13] V. S. Stubican, G. S. Corman, J. R. Hellmann, G. Senft, Advances in Ceramics v. 12, Science and Technology of Zirconia II, ed. N. Claussen, M. Rühle, A. H. Heuer, Am. Ceram. Soc. Columbus, Ohio (1984) p. 96-106 .

[14] D. G. Lamas, R. E. Juarez, A. Caneiro, N. E. Walsöe de Reca, Nanostructured Mater. 10 (1998) 1199.

[15] F. F. Lange, J. Mater. Sci. 17 (1982) 225.

[16] R. E. Juarez, D. G. Lamas, G. E. Lascalea, N. E. Walsöe de Reca, Defects and Diffusion Forum 177-178 (1999) 1.

[17] R. C. Garvie, P. S. Nicholson, J. Am. Ceram. Soc. 55 (1972) 152.

[18] E. N. S. Muccillo, M. Kleitz J. Eur. Ceram. Soc. 16 (1996) 453.

[19] E. N. S. Muccillo, Tese de Doutorado, USP (1993).

[20] A. P. Santos, R. Z. Domingues, M. Kleitz, J. Eur. Ceram. Soc. 18 (1998) 1571.

[21] S. K. Tadokoro, E. N. S. Muccillo, Cerâmica 46 (2000) 230.

[22] H. Qiu, L. Gao, C. Feng, J. Guo, D. Yan, J. Mater. Sci. 30 (1995) 5508.

[23] M. Rühle, N. Claussen, A. H. Heuer, in Advances in Ceramics v. 12, Science and Technology of Zirconia II, ed. N, Claussen, M. Rühle, A. H. Heuer, Am. Ceram. Soc., Columbus, Ohio (1984) p. 352-370.

[24] V. G. Keramidas, W. B. White, J. Am. Ceram. Soc. 57 (1974) 22.

[25] C. Leach, J. Mater. Sci. Lett. 11 (1992) 306.

[26] L. Dessemond, R. Muccillo, M. Hénault, M. Kleitz, Appl. Phys. A57 (1993) 57.

[27] M. Kleitz, L. Dessemond, M. C. Steil, Solid State Ionics 75 (1995) 107.

[28] J. R. Mcdonald, Impedance Spectroscopy-emphasizing solid materials and systems, John Wiley \& Sons (1987) p. 201.

[29] T. H. Etsell, S. N. Flengas, Chem. Rev. 70 (1970) 339. (Rec. 28/11/2005, Ac. 28/02/2006) 